\title{
Upper Extremity Physical Therapy for Stroke Patients using a General Purpose Robot
}

\author{
Hee-Tae Jung, Jennifer Baird, Yu-Kyong Choe, and Roderic A. Grupen
}

\begin{abstract}
In recent years, the potential of robot-mediated upper extremity therapy has been widely acknowledged in stroke rehabilitation. The primary focus of rehabilitation robotics has been on building exoskeleton robots as well as devising control strategies that leverage robotic forces to adaptively assist and resist the movements of patients' impaired arms during repetitive reach-touch exercises. In our single subject case study, a robot presents exercise target positions that are successively more difficult to the patient. This serves to adaptively challenge the patient, while inducing voluntary reach-touch movements. Through data collected during 12 weeks of therapy with a single subject, we demonstrate that a general purpose robot can induce desired therapeutic exercise movements from a patient. In addition, we have observed that the challenge level can be adapted as the patient improves his motor function, which leads to observable improvements in the motor function of the patient.
\end{abstract}

\section{INTRODUCTION}

A stroke is one of the leading causes of death in the world, including the United States [1]. Even if a patient survives from a stroke, often times they are left with permanent disabilities, such as hemiparesis. In order to regain and continue to sustain motor function, patients with a stroke undergo a variety of therapies. These routines are administered by experienced human therapists in conventional rehabilitation. Recent experimental results suggest that patients are able to recover motor function through intensive therapies, even at the chronic phase [2]. Due partially to the substantial economic burden, however, length of stay for inpatient rehabilitation has decreased. As a result, the majority of stroke survivors do not receive a sufficient amount of rehabilitation service [3]. To effectively address this problem, many have proposed adopting robot-mediated therapies [4]-[6].

Despite the variance in design, rehabilitation robots used for upper extremity gross motor exercises are generally built to adaptively challenge patients while providing repetitive therapeutic exercises to them [4]. This is achieved by applying robotic forces that assist or resist the reachtouch $^{1}$ movement of the patients' impaired arms as needed. Randomized control studies suggest that these approaches

H. Jung is with the Laboratory for Perceptual Robotics, Department of Computer Science, University of Massachusetts Amherst (UMass Amherst), MA 01003-9264, USA hjung@ cs . umass . edu

J. Baird is with the Motor Control Laboratory, Department of Kinesiology, UMass Amherst, MA 01003-9258, USA bupt 95 @kin.umass. edu

Y. Choe is with Faculty of Department of Communication Disorders, UMass Amherst, MA 01003-9296 ychoe@comdis.umass.edu

R. A. Grupen is the director of the Laboratory for Perceptual Robotics and is with Faculty of Department of Computer Science, UMass Amherst, MA 01003-9258, USA grupen@ c. . umass . edu

${ }^{1}$ In the literature, it is referred to interchangeably as forward reach, reach, reaching and so on. can cause observable improvement in motor function which is comparable to the improvement achieved from intensive therapy with human therapists [7]. Empirical study suggests that adaptively challenged movement exercises may in fact be one of the primary sources in motor recovery, regardless of the employed therapy medium [8].

Based on these findings, we design a case study which departs from the contemporary approaches to robot-mediated rehabilitation and with this data we support the feasibility of physical therapy using a general purpose robot. In our study, the robot engages a patient as a social medium and induces desired reach-touch movements from the patient through various target positions presented by its hands. The robot chooses target positions that are successively more difficult in order to keep the exercises sufficiently challenging and engaging as the patient improves motor function. The patient reaches for the targets through either self-assisted or unassisted voluntary movements. The data collected during the 12 weeks of therapy suggest that physical therapy through a general purpose robot can provide adaptively challenging therapeutic activities and lead to a motor recovery of a patient with stroke observable by both Fugl-Meyer Assessment $(\text { FMA })^{2}$ [9], [10] and Wolf Motor Function Test (WMFT) ${ }^{3}$ [11], [12].

The remainder of this paper is structured as follows. In Section II, we briefly introduce a stroke and a variety of approaches to robot-mediate upper extremity therapy for patients who have experienced a stroke. In Section III, we visit our study design in detail. In Section IV, we report our experimental results. Section V concludes the paper with a discussion of possible future extensions.

\section{BACKGROUND}

\section{A. Stroke and Physical Therapy}

A stroke, medically termed as a cerebral vascular accident, is a rapid loss of brain cells caused by a disturbance of blood supply to the brain. This lack of blood flow can be caused by different reasons, such as arterial blockage or rupture of an artery. The affected area of the brain is

\footnotetext{
${ }^{2}$ The upper extremity portion of the FMA consists of 32 items that evaluate upper extremity functions of stroke patients. A scale with scores for upper extremity impairment ranges from 0 (no function at all) to 66 (normal function). A subset of FMA components can be used to describe improvements in a subset of upper extremity motor functions.

${ }^{3}$ The WMFT quantifies upper extremity motor ability through fifteen timed and functional tasks. The results report how fast patients achieve the tasks in addition to how many they successfully do. Accordingly, WMFT can measure improvements that cannot be measured in discrete scoring of FMA.
} 
thus unable to function, which leads to deficits that may include difficulty in moving the arm and/or leg, understanding or producing speech and perceiving the environment [13]. To alleviate these impairments, stroke patients often pursue rehabilitation therapy at the acute, sub-acute, and chronic phases as necessary. The goal of physical therapy for individuals who have suffered a stroke is to help them regain functional abilities, such as walking, as close as possible to their abilities before the strokes. The deficits that individuals display are assessed and treated through a variety of tasks that are designed to help them regain strength and use of the affected side. The exercises are often designed to reinforce the activities that are performed in daily life through the repetition of exercise movements prescribed by a therapist. Traditionally, it has been reported that most of the recovery occurs within the first few months after stroke [14][16], and rehabilitation after that has been underappreciated. However, recent experimental results suggest that intensive intervention therapies can lead to significant improvement in motor function even during the sub-acute and chronic phases [2], [17]. Due partially to the time-consuming and laborintensive nature of the therapies, this has led to the ongoing development of robots for therapeutic purposes [18].

\section{B. Robot-Mediated Physical Therapy}

Since the development of MIT-MANUS [19], a variety of robot designs have been constructed and tested for upper extremity rehabilitation [4]. Despite the increasing number of robots developed, however, the fundamental approach is essentially the same. The robots are generally designed to physically support the arm movements of patients during therapeutic exercises using various sensors and actuators [18]. Therapy activities employing this type of robot are mostly reach-touch exercises [20]. During physical exercises, stroke patients either hold on to or place their impaired arm on the exoskeletal components of these robots, which provide assistance for low functioning patients to complete otherwise unattainable movements and provide resistance for high functioning patients to make the tasks more challenging while targets are displayed on computer screens. The therapy activities that we employ in our study are also reach-touch exercises, but we do not provide artificial assistance or resistance using applied robotic forces. Rather, we control the challenge level by balancing the number of target positions drawn from different spatial areas for which a subject requires different levels of effort to reach. The subject is asked to reach and touch these targets through either self-assisted or unassisted voluntary movements through instructions given by the robot.

Alternately, there has been an approach that employs an embodied robot that engages patients in a various ways as a social medium. In one study, the robot provides verbal stimuli that encourages patients to continue the prescribed exercises when they pause during exercises [5]. In this study the robot passively observes the patients' movements and does not physically interact with them. In our work, however, the robot actively engages the patient and leads

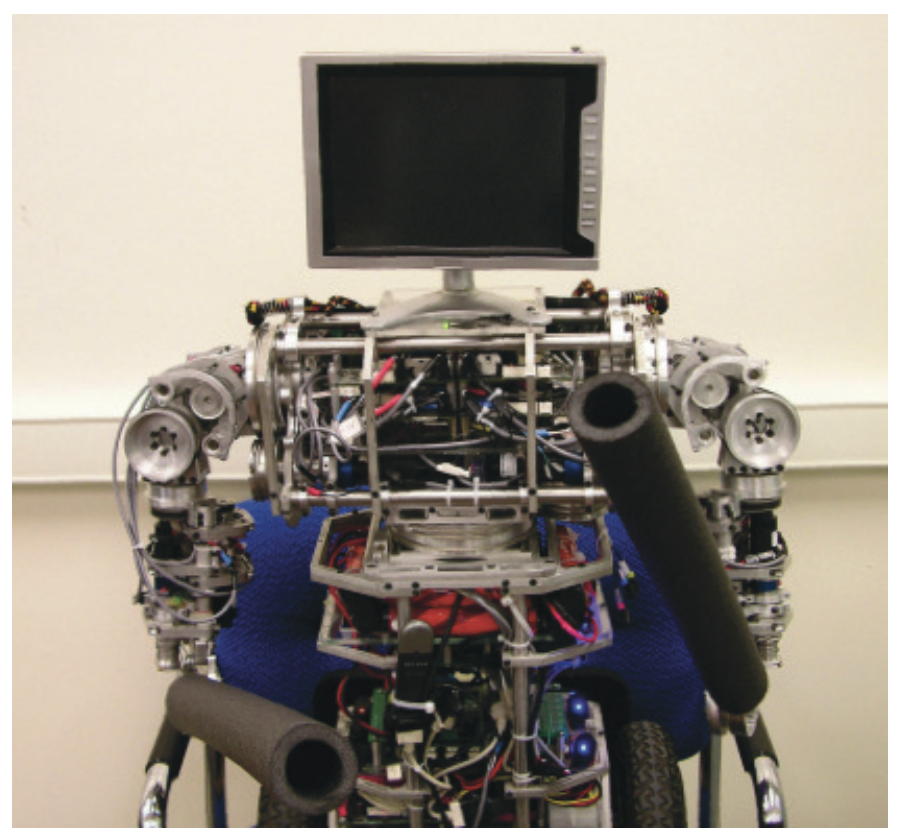

Fig. 1: The uBot-5 is used as the robot platform throughout the study. An LCD monitor is used to display video instructions.

the therapeutic activities. In another study, the robot actively engages the patient during therapy sessions as it does in ours, but the challenge level is not adjusted as the patient regains motor function [6]. Neither of these studies report therapeutic effects [5], [6].

\section{STUDY DESIGN}

\section{A. Human Subject}

We recruit the subject based on the following inclusion criteria. The subject should be at least 18 years old and have had a stroke 6 months or more prior to enrollment. Assessed impairment should be scored between 7 to 38 (out of 66) on the FMA.

The subject in this study was a 72-year-old male with chronic hemiparesis who suffered from a stroke 9.5 years prior to enrollment. He scored 31 (out of 66) on the initial assessment. His score on the relevant items was 24 (out of 52) at the start of the case study. Before joining the study, written consent was obtained.

\section{B. Robot Platform}

The uBot-5, a small light-weight bimanual mobile manipulator developed at the Laboratory for Perceptual Robotics at UMass Amherst [21], delivers physical therapy. To reduce risk from unexpected accidents throughout the therapy sessions, the robot sits across a table and thus the patient is always out of the reach. We use Microsoft Robotics Developer Studio for the software development environment.

\section{Physical Therapy}

During the design of the therapy, a physical therapist is present to provide knowledge on traditional therapies which 


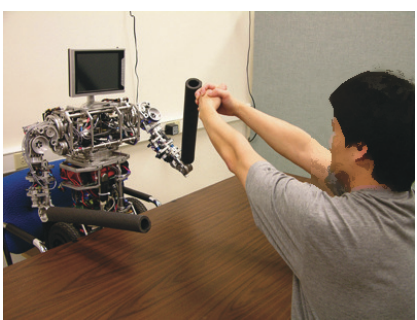

(a) Task 1. Self-assisted two-arm reach-touch.

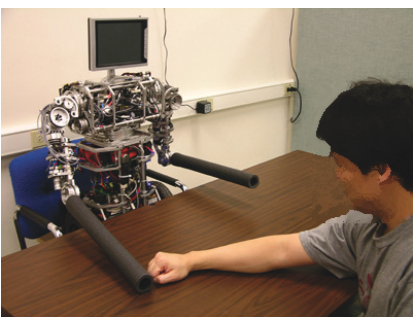

(b) Task 2. Unassisted one-arm sliding reach-touch.
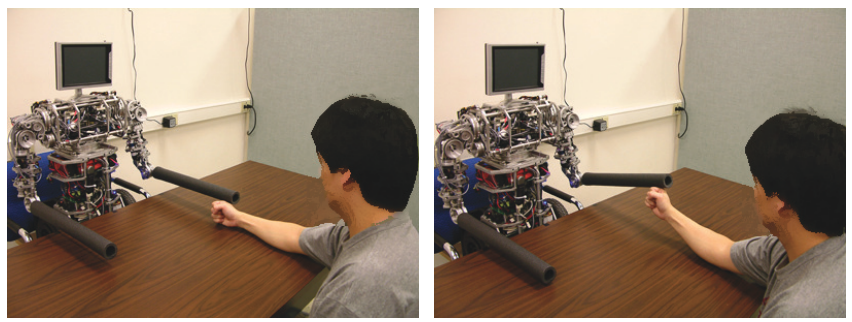

(c) Task 3. Unassisted forearm rotat- (d) Task 3. Unassisted forearm lifting reach-touch.

ing reach-touch.

Fig. 2: The three physical exercise tasks employed in the study, which were prescribed by a human physical therapist.

have proven to be effective. Physical exercise tasks for the subject are determined based on the subject's current level of motor function as well as through discussion between the therapist and the subject. The focus of the robot-mediated physical therapy is on dominant right arm function. The subject engages in the following three therapy exercises three times a week for twelve weeks.

1) Task 1. Holding two hands together and stretching arms to reach for the robot's hand which is presented at various points on the surface of the patient's reachable workspace; See Fig. 2a.

2) Task 2. Flexing and extending the elbow joint to touch the robot hand which is presented at various points on the horizontal plane within the distance of the subject's arm length; See Fig. 2b.

3) Task 3. Rotating or lifting the forearm to touch the robot hand which is presented above the patient's hand; See Fig. 2c and 2d.

Task 1 is intended to induce a large range of motion and to stretch arm joints through self-assisted movements. Task 2 and 3 are intended to induce unassisted voluntary movements. Initially, Task 3 acts to induce wrist rotation movements. As the patient regains his motor function, target positions of Task 3 are provided higher than those he can reach just by rotating his wrist. This naturally induces forearm lifting movements as soon as the patient becomes capable. Throughout the study the target positions are adjusted. The patient is expected to attain the given targets in a way that he feels comfortable with, rather than trying to follow specific arm movement trajectories. When the patient can reach a given target position as instructed by the robot, it is counted as a success. Each task is presented for five minutes with minutes of deviation based on the patient's performance

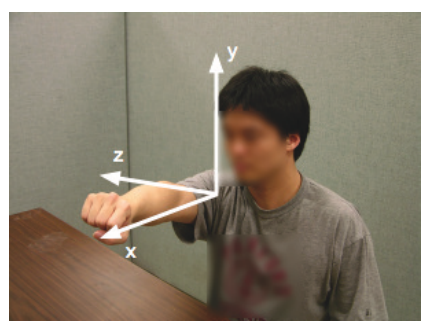

(a) The origin of the frame is at the impaired shoulder of the patient.

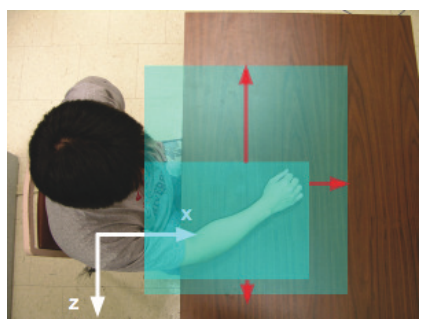

(c) Adjustment for the task 2 is done (d) Adjustment for the task 3 is done in a way that $x$ coordinate of the in a way that $y$ coordinate of the target positions gets larger and the target positions gets larger. absolute value of the $z$ coordinate gets larger.

Fig. 3: Approximate three dimensional description of the controlled directional adjustment of the challenge level for the prescribed tasks.

and fatigue.

\section{Choosing Target Positions}

For the prescribed tasks, we choose the initial sets of target positions which the patient can attain with efforts. These initial sets do not include the positions that the patient cannot reach. Given these sets, the uBot-5 is programmed to choose a random sequence of positions to promote a large range of motion. As the study proceeds, the challenge level of targets are adjusted at the request of the patient or researchers by replacing one or more target positions that the patient can easily attain with more challenging ones. Fig. 3 describes the overall direction of adjustment in each exercise task. When the subject attains the adjusted target positions more than once, they are kept for the following sessions. When the subject cannot attain the adjusted targets at all, the positions are readjusted until the patient can attain them.

\section{E. Therapy Procedure}

Fig. 5 describes the overall therapy procedure. Physical therapy starts with a short greeting. Then the uBot-5 initiates each task in turn by displaying a recorded instruction video on the LCD monitor that is mounted on top of the robot; See Fig. 4a. For instance, before initiating Task 1, the uBot5 displays a video instruction that describes the task; See Fig. 4b. In the video, the therapist says "Clasp your hands together and reach out to touch my hand" and the uBot5 provides the first target position for the two-arm reach- 

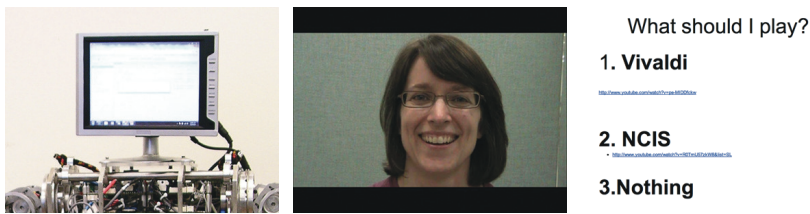

(a) LCD monitor is (b) A recorded instruc- (c) A list of refreshment mounted on the uBot-5. tion on a task.

options.

Fig. 4: Before each exercise task, the recorded instruction and demonstration are displayed on the LCD monitor which is mounted on the uBot-5. During the break between exercises, a short video clip is displayed based on the patient's choice.

touch exercises with its hands. When the patient successfully reaches and touches the uBot-5's hand, the uBot-5 provides the next target position. At completion of a 5-minute exercise, the uBot-5 displays a recorded video clip in which the therapist says "Good job. It is time to take a break." The exercise is followed by approximately five minutes of rest. During the break, a list of refreshment options is provided on the uBot-5's LCD monitor; see Fig. 4c. A short video clip or audio chosen by the patient is played during the break. Task 2 and 3 are done likewise. The exercise Task 1, 2, and 3 are repeated in an order until the patient feels tired and wants to stop.

\section{RESULTS}

\section{A. Task-Specific Progress}

We logged the number of movements and corresponding target positions that the patient successfully made at each session as we made adjustments; see Fig. 6. The target positions that were presented to the patient but not attained were excluded when computing the average. Unlike the previous study [6], we did not see the continuous increase in the number of successful movements since we adjusted the targets to be successively more challenging as the subject improved in motor function. As you can see in Fig. 6b, the subject was able to attain the target positions which were placed higher and farther from the shoulder of the patient as the study proceeded. It the early sessions of the study, on average, the Task 1 targets were presented at the height of the patient's shoulder. However, as you can see in Fig. 6a, he was able to reach the targets with the average of $0.34 \mathrm{~m}$ higher by the last session, which is higher than the top of the patient's head. For Task 2, the patient was able to reach the targets with the average of $0.18 \mathrm{~m}$ farther than he did in the initial session. It is interesting to note that, by Session 16, Task 3 became forearm lifting exercise rather than forearm rotating exercise because of the adjusted challenge level of the target positions and the improved motor function of the patient. In the end, the patient was able to reach target positions located as high as $0.28 \mathrm{~m}$ from the table top while initially the patient was not able to lift his forearm at all. Note that, in Session 14, the therapist provided instructions on proper movements after observing the patient complete several exercises. Thereafter, the subject focused on making correct movements, rather than simply more movements. This was the fundamental

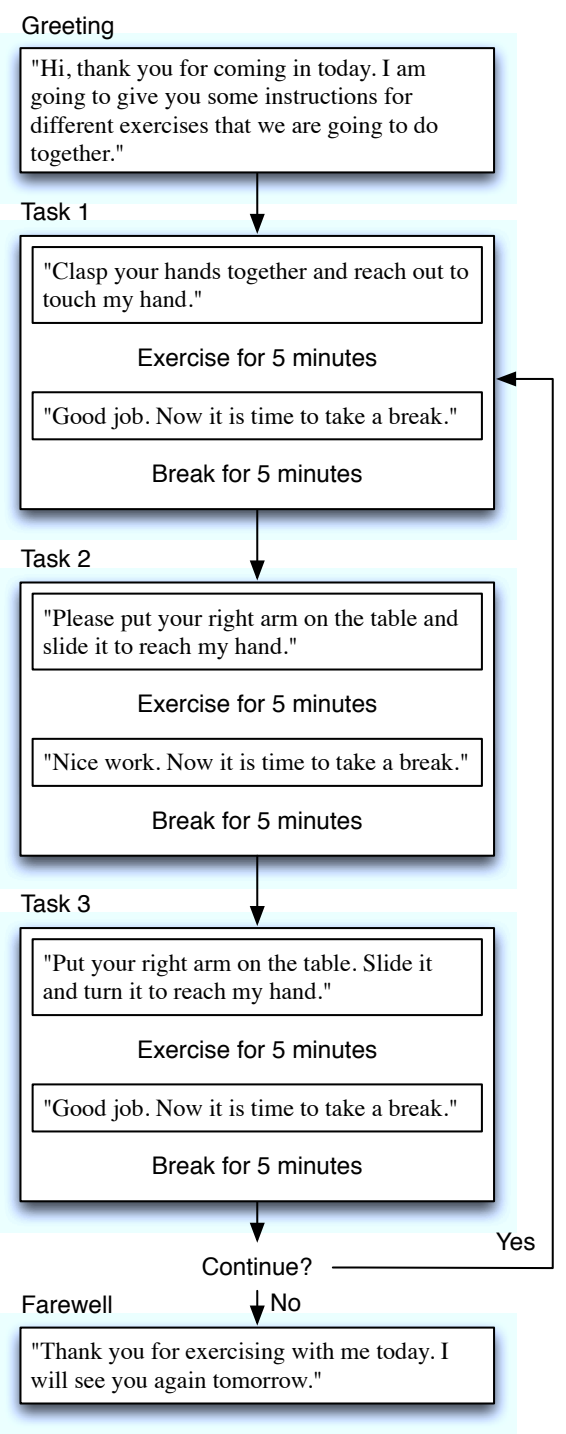

Fig. 5: The therapy procedure and recorded script used during the therapy sessions.

reason for the decrease in the number of movements per minute.

\section{B. Standardized Assessment}

Therapy-induced improvements ware observed in both FMA and WMFT; see TABLE I and II. The subject demonstrated the ability to actively lift his arm higher, as observed in the Flexor Synergy item on the FMA. He was able to lift his arm to his cheek $^{4}$ whereas initially he was able to lift his arm only to chest height. On the WMFT, his time on the three items he did complete decreased from a total of 14.85 seconds to 4.28 seconds and 9.15 seconds after 6 weeks and 12 weeks respectively. In addition, he was able to come closer to completing some of the tasks. For example, he was able to lift his arm almost completely to the top of a target box (item 2 of the WMFT) on the post-treatment test

\footnotetext{
${ }^{4}$ The goal is to lift the arm to the ear.
} 


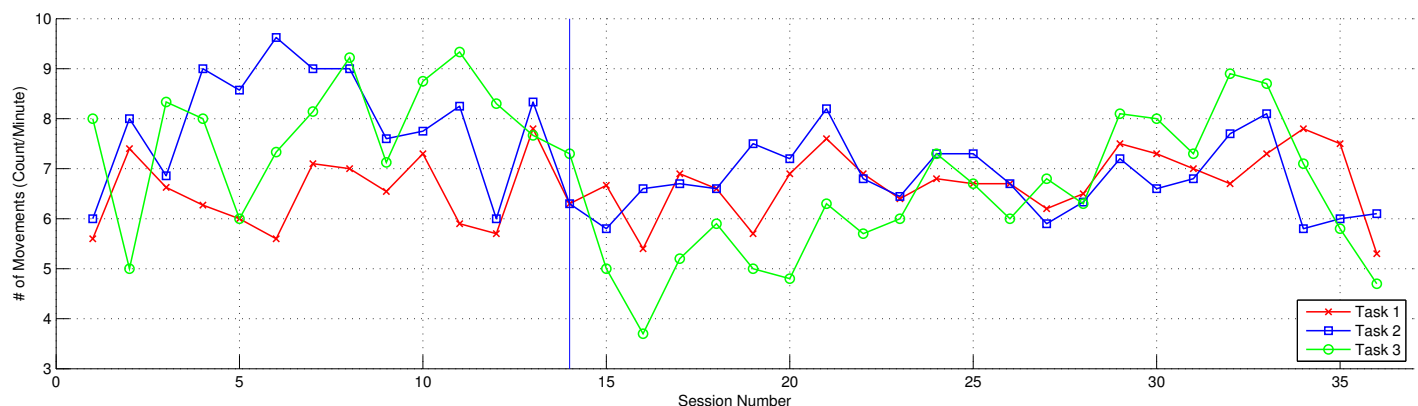

(a) The number of movements per minute throughout the study. The blue vertical line indicates the session in which the therapist provided feedback on the subject's performance.

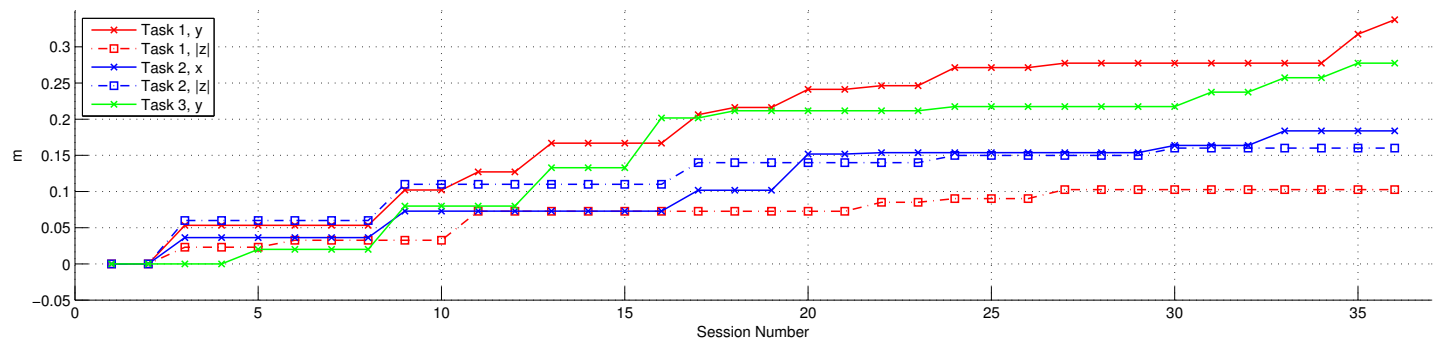

(b) The average increases in $x, y$, and $|z|$ of the target positions attained by the patient in each session throughout the study. Note that the values indicate the relative average increase with respect to the average values in the very first session.

Fig. 6: The patient was able to keep up the number of exercise movements as he regained motor function although the presented target positions became successively more challenging throughout the study. Best viewed in color.

TABLE I: Score changes in FMA (points). The three components (out of total four upper extremity assessment components) that are directly related to the prescribed therapy activities are presented here.

\begin{tabular}{c|ccc}
\hline Component & Baseline & After 6 weeks & After 12 weeks \\
\hline Shoulder/elbow/forearm & $18 / 36$ & $16 / 36$ & $17 / 36$ \\
Wrist & $3 / 10$ & $4 / 10$ & $4 / 10$ \\
Co-ordination/speed & $3 / 6$ & $4 / 6$ & $4 / 6$ \\
\hline Total & $24 / 52$ & $24 / 52$ & $25 / 52$ \\
\hline
\end{tabular}

TABLE II: Time changes in WMFT (seconds). Only the items that the patient were able to complete are presented here.

\begin{tabular}{c|ccc}
\hline Component & Baseline & After 6 weeks & After 12 weeks \\
\hline Forearm to table & 4.03 & 1.90 & 4.12 \\
Hand to table & 5.56 & 1.38 & 2.34 \\
Reach and retrieve & 5.26 & 1.00 & 2.69 \\
\hline Total & 14.85 & 4.28 & 9.15 \\
\hline
\end{tabular}

after 12 weeks of therapy, yet he was able to lift his arm only halfway up the box on the initial test.

These observations indicate that he did demonstrate improvement in motor ability. This result also conforms with the results seen with the tasks he performed with the uBot5 , which were made successively more difficult during the training sessions. Ultimately, it will be important to assess and observe changes in the subject's ability to use his arm for everyday activities.

\section{CONCLUSIONS AND FUTURE WORK}

\section{A. Conclusions}

We envision a future in which robots will reside with humans to provide general purpose services [22]. These services will include healthcare and rehabilitation. Toward that end, we investigated a novel approach to physical rehabilitation for patients with stroke in this study. Departing from the contemporary rehabilitation robotics in which robots are designed to physically support patient's movements during therapy exercises, we employed a general purpose robot which engages a patient by presenting adaptively challenging exercise targets. Instead of providing assisting or resisting robotic forces in order to adjust the challenge level, in our study the robot provides therapeutic exercises by laying out the target positions which require different level of efforts to attain. Our results show that the patient is able to attain target positions higher and farther from his shoulder as the therapy proceeds. This improvement is revealed in standardized assessments as well. This supports that use of general purpose robots in physical therapies has a potential that can complement other contemporary approaches, especially in residential settings. Also, exoskeleton robots are easy to confine the types of therapy activities that can be delivered to patients. However, robots such as the uBot-5 have potential to enable more diverse therapy activities, such as ball rolling or board games, that can induce functional movements that are more useful for everyday activities. 


\section{B. Future Work}

Based on these promising results, we plan to extend the current work in various directions. We intend to establish the efficacy and effectiveness through a large scale experiment involving multiple patients which will produce statistically significant results. Since this is a single subject case study, it is not clear that similar therapeutic outcome will be consistently observed. Also, the comparative therapeutic effects of an embodied agent compared to a simulated agent or a simple game can be studied. A number of empirical study results suggest that the embodiment can be more effective when engaging with humans [5], [23]. Embodiment may be able to achieve longer engagement in physical therapy and, possibly, lead to increased improvement.

Our anecdotal experience suggests that different patients may develop different communicative gestures, even in the same context. During therapy sessions, the subject showed gestures to express his fatigue and confusion. For instance, the subject often looked down at the table after exhausting exercises. When that happened, the subject often failed to perceive a new target position presented by the robot unless it was radically different from the previous one. In these cases, the patient repeatedly looked at the robot's hands one at a time, expressing his confusion. These gestures were not observed with the other subjects of our earlier work. The robot may be able to run therapy sessions more dynamically if it can learn the communicative gestures of patients and respond accordingly.

In this study the strategy of challenge level adjustment during therapy exercise is coarsely programmed by hand. Motor function recovery involves rewiring neural pathways as well as improving muscle strength. Hence, we will need to wield dynamic strategies when adapting the challenge level as patients continue to improve motor function. Using machine learning techniques, it may be possible that we can program the robot to learn more sophisticated therapy strategies from human demonstrations, which are not easily described in words.

\section{ACKNOWLEDGMENTS}

This research was funded in part ONR MURI award N00014-07-1-0749 and the Faculty Research Grant from UMass Amherst to Yu-Kyong Choe. Hee-Tae Jung acknowledges Robin Popplestone Fellowship and Jeong Song Culture Foundation Scholarship. The authors express thanks to Megan Cronin and Jennifer Russell for their help in conducting experiments.

\section{REFERENCES}

[1] V. Roger, A. Go, D. Lloyd-Jones, R. Adams, J. Berry, T. Brown, M. Carnethon, S. Dai, G. de Simone, E. Ford, et al., "Heart disease and stroke statistics-2011 update: A report from the american heart association," Circulation, vol. 123, no. 4, pp. e18-e209, 2011.

[2] W. H. R. Miltner, H. Bauder, M. Sommer, C. Dettmers, and E. Taub, "Effects of constraint induced movement therapy on patients with chronic motor deficits after stroke: A replication," Stroke, vol. 30, pp. 586-592, 1999.

[3] B. Dobkin, "The economic impact of stroke," Neurology, vol. 45, no. 2 Suppl 1, pp. S6-S9, 1995.
[4] P. S. Lum, D. J. Reinkensmeyer, R. Mahoney, W. Z. Rymer, and C. G. Burgar, "Clinical considerations in the use of robotic devices for movement therapy following stroke," Topics in Stroke Rehabilitation, vol. 8, no. 4, pp. 40-53, 2002

[5] M. J. Matarić, J. Eriksson, D. J. Feil-Seifer, and C. J. Winstein, "Socially assistive robotics for post-stroke rehabilitation," International Journal of NeuroEngineering and Rehabilitation, vol. 4, no. 5, 2007.

[6] H. Jung, J. Baird, Y. Choe, and R. A. Grupen, "Upper-limb exercises for stroke patients through the direct engagement of an embodied agent," in Proceedings of the 6th ACM/IEEE International Conference on Human-Robot Interaction, Lausanne, Switzerland, March 2011, pp. $157-158$.

[7] A. C. Lo, P. D. Guarino, L. G. Richards, J. K. Haselkorn, G. F. Wittenberg, D. G. Federman, R. J. Ringer, Pharm.D., T. H. Wagner, H. I. Krebs, B. T. Volpe, J. Christopher T. Bever, D. M. Bravata, P. W Duncan, B. H. Corn, A. D. Maffucci, S. E. Nadeau, S. S. Conroy, J. M. Powell, G. D. Huang, and P. Peduzzi, "Robot-assisted therapy for long-term upper-limb impairment after stroke," The New England Journal of Medicine, vol. 362, no. 19, pp. 1772-1783, 2010.

[8] L. Kahn, P. Lum, W. Rymer, and D. Reinkensmeyer, "Robot-assisted movement training for the stroke-impaired arm: Does it matter what the robot does?" Journal of Rehabilitation Research and Development, vol. 43, no. 5, pp. 619-630, 2006.

[9] A. Fugl-Meyer, L. Jääskö, I. Leyman, S. Olsson, and S. Steglind, "The post-stroke hemiplegic patient: a method for evaluation of physical performance," Scandinavian journal of rehabilitation medicine, vol. 7, no. 1, pp. 13-31, 1975 .

[10] A. Deakin, H. Hill, and V. M. Pomeroy, "Rough guide to the fuglmeyer assessment upper limb section," Physiotherapy, vol. 89, no. 12, pp. 751-763, 2003.

[11] S. L. Wolf, D. E. Lecraw, L. A. Barton, and B. B. Jann, "Forced use of hemiplegic upper extremities to reverse the effect of learned nonuse among chronic stroke and head injured patients," Experimental Neurology, vol. 104, pp. 125-132, 1989.

[12] S. L. Wolf, P. A. Catlin, M. Ellis, A. L. Archer, B. Morgan, and A. Piacentino, "Assessing wolf motor function test as outcome measure for research in patients after stroke," Stroke, vol. 32, pp. 1635-1639, 2001

[13] G. A. Donnan, M. Fisher, M. Macleod, and S. M. Davis, "Stroke," The Lancet, vol. 371, no. 9624, pp. 1612-1623, May 2008

[14] C. E. Skilbeck, D. T. Wade, R. L. Hewer, and V. A. Wood, "Recovery after stroke," Journal of Neurology, Neurosurgery \& Psychiatry, vol. 46, pp. 5-8, 1983.

[15] M. L. Dombovy, J. R. Basford, J. P. Whisnant, and E. J. Bergstralh, "Disability and use of rehabilitation services following stroke in rochester, minnesota, 1975-1979,"' Stroke, vol. 18, pp. 830-836, 1987.

[16] L. Ferrucci, S. Bandinelli, J. M. Guralnik, M. Lamponi, C. Bertini, M. Falchini, and A. Baroni, "Recovery of functional status after stroke. a postrehabilitation follow-up study," Stroke, vol. 24, pp. 200-205, 1993.

[17] J. Liepert, H. Bauder, W. H. R. Miltner, E. Taub, and C. Weiller, "Treatment-induced cortical reorganization after stroke in humans," Stroke, vol. 31, pp. 1210-1216, 2000.

[18] L. Marchal-Crespo and D. J. Reinkensmeyer, "Review of control strategies for robotic movement training after neurologic injury," International Journal of NeuroEngineering and Rehabilitation, vol. 6 , no. 20, 2009

[19] H. I. Krebs, N. Hogan, M. L. Aisen, and B. T. Volpe, "Robot-Aided Neurorehabilitation," IEEE Transactions of Rehabilitation Engineering, vol. 6, no. 1, pp. 75-87, 1998.

[20] G. Kwakkel, B. Kollen, and H. Krebs, "Effects of robot-assisted therapy on upper limb recovery after stroke: a systematic review," Neurorehabilitation and Neural Repair, vol. 22, no. 2, pp. 111-121, 2008

[21] P. Deegan, B. Thibodeau, and R. A. Grupen, "Designing a selfstabilizing robot for dynamic mobile manipulation," in Workshop on Manipulation for Human Environments, Robotics: Science and Systems, Philadelphia, Pennsylvania, August 2006.

[22] P. Deegan, R. A. Grupen, A. Hanson, E. Horrell, S. Ou, E. Riseman, S. Sen, B. Thibodeau, A. Williams, and D. Xie, "Mobile manipulators for assisted living in residential settings," Autonomous Robots, vol. 24, no. 2, pp. 179-192, 2007.

[23] W. Bainbridge, J. Hart, E. Kim, and B. Scassellati, "The Benefits of Interactions with Physically Present Robots over Video-Displayed Agents," International Journal of Social Robotics, pp. 1-12, 2011. 